\title{
Patients assisted at the Department of Medicine of a pediatric hospital at the beginning of the COVID-19 pandemic in Buenos Aires, Argentina
}

\author{
Héctor Cairoli, M.D. ${ }^{a}$, Silvina Raiden, M.D. ${ }^{a}$, María J. Chiolo, M.D. ${ }^{b}$, Sandra Di Lalla, M.D. ${ }^{c}$ and \\ Fernando Ferrero, M.D. ${ }^{a}$ \\ Collaborators: José Blanco Vitorero, M.D., Edgardo Checacci, M.D., Carolina Davenport, M.D., \\ Paula Dominguez, M.D., Horacio Planovsky, M.D., Fabián Gambarruta, M.D., Mariano Ibarra, M.D., \\ María Fabiana Ossorio, M.D., Javier Potasnik, M.D., Norma Schenone, M.D., Milagros Torrents M.D., and \\ Fernando Torres M.D.
}

\begin{abstract}
Introduction. Despite the magnitude of the COVID-19 pandemic, theinformation about its development in pediatricsis still limited. This report describes the characteristics of patients admitted to a children's hospital due to COVID-19 during the first three months of the pandemic.

Method. Descriptive study including all patients hospitalized due to COVID-19 between 4/1/2020 and 6/30/2020.

Results. A total of 191 patients were hospitalized due to COVID-19; their median age was 7.7 years; $89 \%$ had a history of close contact. Of them, $35.6 \%$ were considered asymptomatic; $61.2 \%$, mild cases; and $3.2 \%$, moderate cases (no severe cases). None of them received a specific treatment for the disease. The most common symptoms were fever, sore throat, and cough. The median length of stay was 6 days.

Conclusion. A total of 191 cases of children and adolescents admitted due to COVID-19 are reported. Most were asymptomatic or presented with a mild disease.

Key words: coronavirus infections, COVID-19, pneumonia.
\end{abstract}

http: / / dx.doi.org/ 10.5546/ aap.2020.eng.423

To cite: Cairoli H, Raiden S, Chiolo MJ, Di Lalla S, Ferrero F. Patients assisted at the Department of Medicine of a pediatric hospital at the beginning of the COVID-19 pandemic in Buenos Aires, Argentina. Arch Argent Pediatr 2020; 118(6):423-426.

\section{INTRODUCTION}

In just a few months, the disease now known as COVID-19 had a global distribution and caused millions of cases and hundreds of thousands of deaths worldwide. ${ }^{1}$ Although information is still limited, this pandemic would seem to be less severe in the pediatric population, since most of the described cases present with few symptoms, a favorable course, and low morbidity and mortality. ${ }^{2}$

A timely report made in China indicated that only $5.9 \%$ of pediatric cases were deemed severe. ${ }^{3}$ Further information coming from Europe seems to confirm this initial estimation. ${ }^{4}$

Given that the development of the pandemic seems to have some kind of relation with socioeconomic and cultural variables, it may have specific characteristics in each country. ${ }^{5}$ In Argentina, there is still little information on the development of the pandemic in pediatrics. The objective of this study is to describe the characteristics of patients admitted to a children's hospital in relation to COVID-19 during the first three months of the pandemic.

\section{POPULATION AND METHODS \\ Design: Descriptive study.}

Population: Patients hospitalized in relation with the COVID-19 pandemic in specific areas of Hospital General de Niños Pedro de Elizalde which were designated for this purpose, between $4 / 1 / 2020$ and $6 / 30 / 2020$.

This population included COVID-19 patients who were screened at the hospital's Emergency Department, referred from different health facilities with a confirmed diagnosis, and suspected cases awaiting test results. Patients with other conditions who required hospitalization and were considered suspected cases, either before admission or during their stay at the hospital, were also included. 
The COVID-19 designated ward included an area for suspected cases with strict individual isolation and two areas for confirmed cases with the ability to group them into cohorts and provide them comprehensive care because of this disease and/or an underlying condition.

Study outcome measures: In all cases, severe acute respiratory syndrome coronavirus 2 (SARS$\mathrm{CoV}$-2) screening results, through real-time polymerase chain reaction (RT-PCR), sex, age, place of residence, epidemiological history (close contact with a confirmed case), presence of comorbidities, and length of stay were recorded. Severity was assessed based on the description made by Dong. ${ }^{3}$

Statistical analysis: Categorical outcome measures were described using percentages with $95 \%$ confidence intervals (95 CI), and continuous outcome measures were expressed as median and interquartile range (IQR), according to distribution (Kolmogorov-Smirnov). The IBM SPSS Statistics 20.0 software was used for data analysis.

Ethical considerations: The approval of the hospital's Board and Research Ethics Committee was requested and obtained.

\section{RESULTS}

In the study period, 601 children and adolescents were admitted to the COVID-19 designated ward, who were accompanied by 516 adults in charge. The number of hospitalizations per week ranged from 11 to 84 (Figure 1).

In $31.8 \%$ of children and $28.5 \%$ of caregivers, the presence of SARS-CoV-2 was confirmed by PCR. Of the patients in whom the disease was ruled out $(n=410), 80.7 \%$ were timely discharged and $19.3 \%$, transferred to another area (noCOVID) of the hospital because of their condition (e.g., patients with cancer or requiring surgery).

The median age of the 191 patients with COVID-19 was 7.7 years (IQR: 1.7-13.1). Of them, $53.9 \%$ were males. The place of residence of $85.3 \%$ of COVID-19 patients was located within the hospital's jurisdiction (Autonomous City of Buenos Aires, CABA); and, of them, $41.1 \%$ lived in a poor neighborhood. History of close contact with COVID patients was observed in $89 \%$. Comorbidities were seen in $27.7 \%$; asthma being the most common one $(5.8 \%)$ (Table 1$)$.

Regarding clinical presentation, $35.6 \%$ were asymptomatic; $61.2 \%$, mild cases; and $3.2 \%$, moderate cases. No severe or critical cases were observed. ${ }^{8}$ None of the patients received a specific treatment for the disease. The most common initial symptom was fever, followed by sore throat and cough (Table 2).

The median length of stay was 6 days (IQR: 4-9). At the time of this report, COVID-19

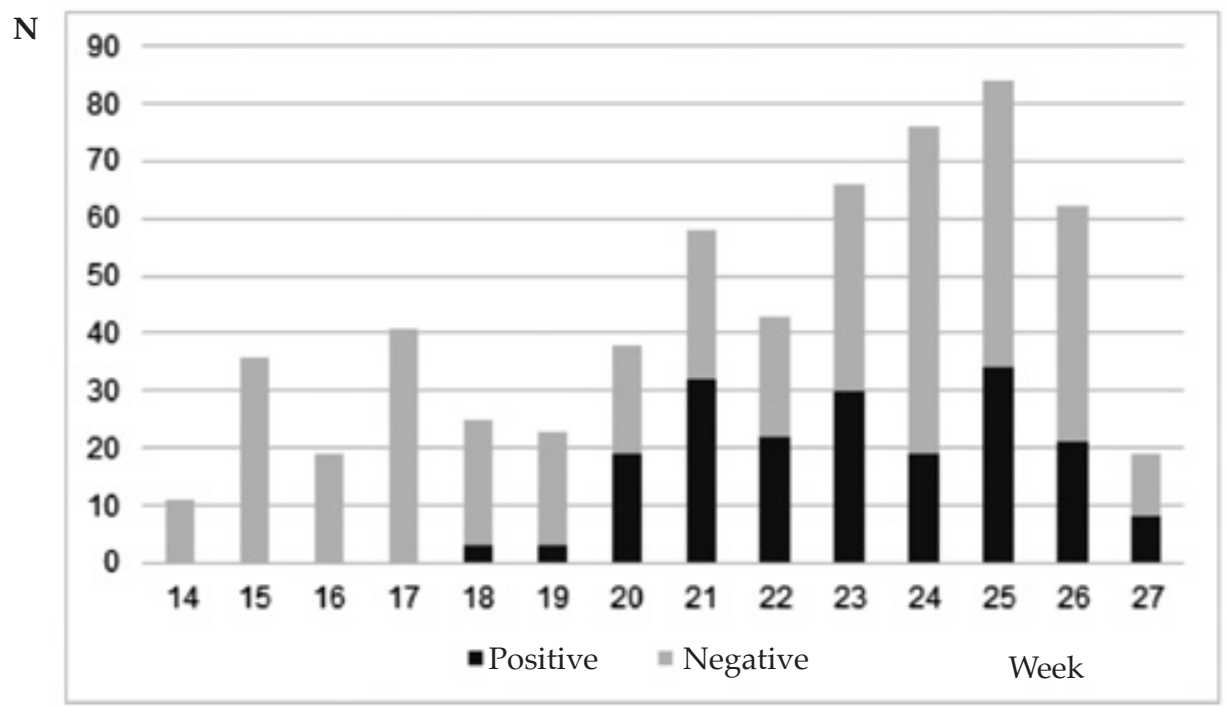

(Week 27, ending on 7/5/2020, only includes cases admitted up to 6/30/2020). 
patients had been discharged without complications (with phone follow-up) or were still hospitalized for treatment, if their underlying disease required it.

\section{DISCUSSION}

This study reports our initial experience caring for pediatric patients with COVID-19. A total of 191 patients received care in the first three months of the pandemic in Argentina.

In the same period, CABA reported 32806 cases; of these, $4719(14.4 \%)$ corresponded to patients younger than 18 years. ${ }^{6}$ Reports from China indicated that $2.1 \%$ of COVID-19 cases corresponded to children and adolescents. ${ }^{7}$ In Italy, $1.2 \%$ of cases were reported to occur in this age group, ${ }^{8}$ and in the USA, up to April, $1.7 \%$ of cases had been reported to correspond to children and adolescents. ${ }^{9}$ Although this point is still under discussion, the age structure and social habits of different populations may have an impact on the observed differences. ${ }^{10}$ It is worth recalling that in Argentina, for instance, the population younger than 15 years almost doubles that of Italy (as per the data of the World Bank, $25 \%$ versus $13 \%$ ).

There are also differences in the proportion of children and adolescents requiring hospitalization. A European multicenter study that included 582 children with SARSCoV-2 infection reported that $62 \%$ required hospitalization. ${ }^{11}$ The Centers for Disease Control and Prevention (CDC) reported an overall hospitalization rate due to COVID-19 of 102.5 per 100000 population in the US, in which 8.9 corresponded to patients aged $0-4$ years and 4.0 , to patients aged 5-17 years. ${ }^{12}$

TABLE 1. Comorbidities in pediatric patients hospitalized with COVID-19 $(n=26)$

\begin{tabular}{ll}
\hline Diagnosis or history & $\mathbf{n}$ \\
\hline Asthma & 12 \\
Diabetes mellitus & 2 \\
Chronic encephalopathy & 2 \\
Medulloblastoma & 2 \\
Obesity & 2 \\
Hemorrhagic stroke & 1 \\
Herpes zoster & 1 \\
Convulsive syndrome & 1 \\
Chronic kidney injury & 1 \\
Systemic lupus erythematosus & 1 \\
Thrombocytopenic purpura & 1 \\
\hline
\end{tabular}

In addition, the results of our study may have been affected by other factors. On the one side, the systematic screening carried out in the pediatric population of neighborhoods with high community transmission of the virus probably increased the proportion of infected children, even though most of them were asymptomatic. On the other side, a considerable part of the population seen at our hospital came from poor neighborhoods and did not meet the required conditions for out-of-hospital isolation of asymptomatic cases, and were therefore hospitalized. ${ }^{13}$ Finally, the fact that, since the end of May, our institution decided to systematically screen every patient requiring hospitalization for other reasons than COVID-19 may have also had implications.

Regarding the severity of the disease in the pediatric population, our experience also found that most cases were mild or asymptomatic. Only 6 moderate cases were observed, and no severe/ critical ones. In China, Dong et al., reported that $51 \%$ of cases were mild; $38.7 \%$, moderate; and $5.9 \%$, severe / critical. ${ }^{3}$ In Spain, Tagarro et al., indicated that only $9.7 \%$ of the cases in their series were severe. ${ }^{4}$ The European multicenter study carried out by Götzinger et al., showed that $9 \%$ required intensive care and $16 \%$ were asymptomatic. ${ }^{11}$

As mentioned above, we should be cautious when comparing this point with our series. Our experience may reflect a higher proportion of mild or asymptomatic patients, given that the Ministry of Health of the CABA adopted a strategy of active search of cases in the community, which is why several of our patients were identified while studying close contacts.

TABLE 2. Clinical presentation of hospitalized patients with COVID-19 $(n=191)$

\begin{tabular}{llcc}
\hline & Symptom & $\mathbf{n}$ & $\%$ \\
\hline Asymptomatic & & 68 & 35.6 \\
Symptomatic $^{*}$ & & & \\
& Fever & 55 & 27.2 \\
& Sore throat & 23 & 12 \\
& Cough & 20 & 10.5 \\
& Headache & 14 & 7.3 \\
& Rhinorrhea & 11 & 5.7 \\
& Abdominal symtoms & 6 & 3.1 \\
& Anosmia & 5 & 2.6 \\
& Dysgeusia & 2 & 1 \\
& Rash & 1 & 0.5 \\
\hline
\end{tabular}

*Initial symptom (patients could have more than one symtom.) 
Fever was the most common symptom, followed by upper respiratory tract symptoms and headache, similar to what has been reported by Götzinger et al. ${ }^{11}$ At the time of this report, no cases of Kawasaki-like multisystem inflammatory syndrome related to COVID-19 (MIS-C) were recorded. Nevertheless, it has been reported that the onset of this syndrome usually occurs weeks after the peak of pediatric cases, which seemed not to have been reached yet. ${ }^{14}$ Asthma was the most common comorbidity, similar to what has been reported. ${ }^{8}$

The progression in the number of hospitalizations somehow reflects the development of the disease in the CABA. It also clearly shows the moment when the Ministry of Health of the CABA started actively looking for cases in poor neighborhoods. This strategy was adopted during week 19; since then, not only the number of hospitalizations in our facility but also the proportion of patients with PCRconfirmed SARS-CoV-2 infection increased. ${ }^{15}$

A limitation of this study is that it includes only a case series from a single site. Nevertheless, at the time of writing this report, our hospital was the public health institution who had hospitalized more pediatric patients with COVID-19 in the CABA.

It is also important to highlight that, as with all the information regarding this pandemic, we should be very cautious when interpreting it because of the novelty of the situation. We should wait for the results of extensive epidemiological studies in order to get a clearer picture of the disease.

\section{CONCLUSION}

This study reported 191 cases of children and adolescents hospitalized due to COVID-19. Most were asymptomatic or presented with a mild form of the disease.

\section{Acknowledgments}

We would like to thank:

- The Emergency Department and its Emergency Fever Unit for the initial care of patients.

- The Central Lab and its Area of Molecular Biology for performing the diagnostic tests since the early stages of the pandemic.

- Pediatric residents, who undertook their tasks with enthusiasm and commitment.

- Valeria Aprea, M.D., coordinator of the Crisis Committee of Hospital General de Niños Pedro de Elizalde, for leading the hospital's response to this situation.

\section{REFERENCES}

1. World Health Organization, Coronavirus Disease 2019 (COVID-19) Situation Report - 140 (WHO, 2020). [Accessed on: June $\left.8^{\text {th }}, 2020\right]$. Available at: https:// www.who.int/ docs/default-source/coronaviruse/ situation-reports / 20200608-covid-19-sitrep-140.pdf? sfvrsn=2f310900_2.

2. Ludvigsson JF. Systematic review of COVID-19 in children shows milder cases and a better prognosis than adults. Acta Paediatr. 2020; 109(6):1088 $\square 95$.

3. Dong $\mathrm{Y}, \mathrm{Mo}$ X, Hu Y, Qi X, et al. Epidemiology of COVID-19 Among Children in China. Pediatrics. 2020; 145(6):e20200702.

4. Tagarro A, Epalza C, Santos M, Sanz-Santaeufemia FJ, et al. Screening and Severity of Coronavirus Disease 2019 (COVID-19) in Children in Madrid, Spain. JAMA Pediatr. 2020;e201346. [Epub, April 8 ${ }^{\text {th }}, 2020$ ].

5. Bong CL, Brasher C, Chikumba E, McDougall R, et al. The COVID-19 Pandemic: Effects on Low and Middle-Income Countries. Anesth Analg. 2020; 131(1):86-92.

6. Argentina. Ministerio de Salud, Gobierno de la Ciudad Autónoma de Buenos Aires. Actualización de los casos de coronavirus en la Ciudad. [Accessed on: July $3^{\text {rd }}$, 2020]. Available at: https://www.buenosaires.gob.ar/ coronavirus / noticias / actualizacion-de-los-casos-decoronavirus-en-la-ciudad-buenos-aires.

7. The Novel Coronavirus Pneumonia Emergency Response Epidemiology Team. The Epidemiological Characteristics of an Outbreak of 2019 Novel Coronavirus Diseases (COVID-19)-China, 2020. China CDCWeekly. 2020;2(8):11322.

8. Livingston E, Bucher K. Coronavirus Disease 2019 (COVID-19) in Italy. JAMA. 2020; 323(14):1335.

9. CDC COVID-19 Response3 Team. Coronavirus Disease 2019 in Children - United States, February 12-April 2, 2020. MMWR Morb Mortal Wkly Rep. 2020; 69(14):422-6.

10. Dowd JB, Andriano L, Brazel DM, Rotondi V, et al. Demographic science aids in understanding the spread and fatality rates of COVID-19. Proc Natl Acad Sci U S A. 2020; 117(18):9696-8.

11. Götzinger F, Santiago-García B, Noguera-Julián A, Lanaspa $\mathrm{M}$, et al. COVID-19 in children and adolescents in Europe: a multinational, multicentre cohort study. Lancet Child Adolesc Health. 2020;S2352-4642(20)30177-2. [Epub, June $\left.25^{\text {th }}, 2020\right]$.

12. CDC. COVID View. A Weekly Surveillance Summary of U.S. COVID-19 Activity. (for Week 26, ending June 27, 2020). [Accessed on: July $\left.3^{\text {rd }}, 2020\right]$. Available at: https:// www.cdc.gov/ coronavirus/2019-ncov/ covid-data/pdf/ covidview-07-03-2020.pdf.

13. Argentina. Ministerio de Salud, Gobierno de la Ciudad Autónoma de Buenos Aires. Manejo frente a casos sospechosos y confirmados de coronavirus (COVID 19). Versión 32. 19 de junio de 2020:16.

14. Capone CA, Subramony A, Sweberg T, Scheider J, et al. Characteristics, Cardiac involvement, and Outcomes of Multisystem Inflammatory Disease of Childhood (MIS-C) Associated with SARS-CoV-2 Infection. J Pediatr. 2020; S0022-3476(20)30746-0. [Epub, June 14 ${ }^{\text {th }}, 2020$ ].

15. Argentina. Ministerio de Salud, Gobierno de la Ciudad Autónoma de Buenos Aires. Dispositivo DetectAr en los barrios. [Accessed on: June $\left.22^{\text {nd }}, 2020\right]$. Availableat: https: / / www.buenosaires.gob.ar/coronavirus/dispositivodetectar. 